\title{
Does BMP2 play a role in the pathogenesis of equine degenerative suspensory ligament desmitis?
}

\author{
Madeline Young ${ }^{1}$, Olaniyi Moshood ${ }^{1}$, Jian Zhang ${ }^{1}$, Carolyn A. Sarbacher ${ }^{1}$, P. O. Eric Mueller ${ }^{2}$ \\ and Jaroslava Halper ${ }^{3^{*}}$ (D)
}

\begin{abstract}
Objective: Horses afflicted with degenerative suspensory ligament desmitis (DSLD) suffer from progressive leg pain and lameness without history of trauma. DSLD is a systemic disorder caused by abnormal accumulation of proteoglycans in many connective tissues. One proteoglycan found in higher quantities in DSLD is decorin. The accumulated decorin has an abnormally glycosylated glycosaminoglycan chain in DSLD. In addition to acellular accumulations of proteoglycans foci of active fibroblasts/tenoblasts were observed in some tendons and suspensory ligaments (SLS) from DSLD cases We have hypothesized that this represents an early event in DSLD and that production of chondrogenic growth factors, such as BMP2, and/or enzyme participating in glycosylation of glycosaminoglycans is a major factor in initiation and progression of DSLD.

Results: Using immunohistochemistry we have identified BMP2 in these cellular foci, indicating association with proteoglycan production, but not in other cells in the tendon and SLs. In contrast, very little staining for TGF $\beta$ and dermatan sulfate epimerase, an enzyme involved in glycosylation of glycosaminoglycan chains, was observed in these foci and other cells in both control and DSLD-affected tendons and SLs. Our data support our hypothesis that chondrogenic growth factors may be responsible, at least in part for progression of DSLD in horses.
\end{abstract}

Keywords: Equine degenerative suspensory ligament desmitis, Proteoglycans, BMP2

\section{Introduction}

Equine Degenerative Suspensory Ligament Desmitis (DSLD) was identified first in Peruvian Paso horses [1]. Though this breed is most commonly affected, DSLD has been diagnosed with increasing frequency in other horse breeds including Quarter horses, Warmbloods, Arabians and others [2, 3]. Affected horses present typically with progressive bilateral or quadrilateral lameness, dropped fetlocks and painful lower extremities with no evidence or history of trauma. The diagnosis of DSLD is based on health history and presentation, pain upon palpation of the suspensory ligament (SL) and diffuse enlargement of

\footnotetext{
*Correspondence: jhalper@uga.edu

${ }^{3}$ Department of Pathology, College of Veterinary Medicine, AU/UGA Medical Partnership, The University of Georgia, Athens, GA 30602, USA

Full list of author information is available at the end of the article
}

SL on physical examination. Ultrasonography of the limb reveals SL enlargement with a hypoechoic, irregular fiber pattern. There is no cure for the disease and care involves supportive and palliative measures such as controlled exercise, corrective shoeing and pain relief [4]. There is no known way to stop the progression of DSLD with many of these horses requiring humane euthanasia. Little is known about the specific pathogenesis of DSLD making prevention, diagnosis and effective treatment of the affected horses difficult. Hereditary component has been suspected because of frequent familial appearance of DSLD during a common age for breeding, the potential for transmitting DSLD to offspring is high [2-4].

Though considered originally a disorder of collagen limited to SLs of lower extremities, later on we characterized DSLD as a systemic disorder caused by abnormal accumulation of proteoglycans in a wide variety of 
connective tissues [3]. This accumulation is particularly damaging to weight-bearing tendons and ligaments of equine extremities [5]. Our lab was the first to bring attention to proteoglycan abnormalities as a potential mechanism $[3,5]$. At least one of the tendon/ligament proteoglycans, decorin, is abnormal in horses with DSLD, with chondroitin sulfate replacing the normal dermatan sulfate due to a defect in glycosylation of the glycosaminoglycan (GAG) chain [5]. Decorin plays an essential role in regulating collagen fibrillogenesis, and spatial organization of collagen fibrils and any abnormality in decorin could lead to abnormal collagen fibrils resulting in decreased biomechanical strength [6-8].

As part of our ongoing study of pathogenesis of DSLD we examined the presence of a chondrogenic growth factor(s) in DSLD using immunohistochemistry. The best characterized chondrogenic factors are transforming growth factor $\beta-3$ (TGF $\beta-3)$, and bone morphogenetic proteins 2 and 7 (BMP2 and BMP7) [9-11]. In addition, of the many enzymes involved in synthesis of GAG chains attached to core proteins of proteoglycans that may play another role in the development of DSLD dermatan sulfate epimerase is of particular interest because of its role in synthesis of dermatan sulfate chains [12-14].

\section{Main text \\ Methods \\ Subjects and tissue collection}

Superficial and deep digital flexor tendons, and SLs were collected from extremities of horses with and without DSLD (Table 1). The horses were either donated for research and euthanized, or were undergoing necropsy for diagnostic purposes. Euthanasia was performed in accordance with institutional guidelines and consisted of intravenous overdose of sodium pentobarbital solution at a dose of $>87 \mathrm{mg} / \mathrm{kg} / \mathrm{IV}$. Some tendon samples were submitted to us for diagnostic purposes.

Table 1 Subject characterization and results of immunohistochemistry

\begin{tabular}{|c|c|c|c|c|c|c|}
\hline Case & Tissue & BMP2 cell & BMP PG & Epimerase & TGF $\beta$ & Age, breed, sex \\
\hline $\mathrm{H} 1$ & SDFT, SL & + & $N / A$ & ++ & $+/-$ & 9 year PP male \\
\hline $\mathrm{H} 2$ & SDFT, SL & $++/+$ & $\mathrm{N} / \mathrm{A}$ & + & - & 15 year PP mare \\
\hline $\mathrm{H} 3$ & $2 \mathrm{SLS}$ & ++ & N/A & $+1-$ & $+/-$ & 18 year $\mathrm{QH}$ mare \\
\hline $\mathrm{H} 4$ & SDFT, SL & ++ & $\mathrm{N} / \mathrm{A}$ & - & ND & 1.5 year PP male \\
\hline $\mathrm{H} 5$ & $\mathrm{SL}$ & ++ & + & $-/+$ & ND & 7 year PP mare \\
\hline $\mathrm{H} 6$ & SDFT, DDFT & $++/+$ & - & $++/+$ & ND & 5 year PP male \\
\hline $\mathrm{H7}$ & SDFT & $++++/+++$ & + & ++ & $+/-$ & 17 year WB gelding \\
\hline $\mathrm{H} 8$ & $2 \mathrm{SLS}$ & ++++ & $+/++$ & $+1-$ & + & 4 year Arab gelding \\
\hline H9 & SFDT, DDFT, SL & $+++/++$ & + & $+1-$ & $+/-$ & 6 year Amer Saddle male \\
\hline $\mathrm{H} 10$ & $\mathrm{SL}$ & - & $++/-$ & $+/-$ & - & Unknown \\
\hline $\mathrm{H} 11$ & $\mathrm{SL}$ & + & + & $-/+$ & ++ & 11 year $\mathrm{QH}$ mare \\
\hline $\mathrm{H} 12$ & SDFT & +++ & - & ++ & - & 9 year $\mathrm{QH}$ gelding \\
\hline $\mathrm{H} 13$ & $\begin{array}{l}2 \text { SDFTS } \\
\text { DDFT, SL }\end{array}$ & $\begin{array}{l}++/+ \\
+/-\end{array}$ & ND & $\begin{array}{l}++ \\
+/-\end{array}$ & ND & 16 year PP female \\
\hline $\mathrm{H} 14$ & $\mathrm{SL}$ & +++ & $++/+$ & $+/-$ & ND & 15 year holsteiner gelding \\
\hline D1 & DDFT, SL & ++ & $+/-$ & $+/-$ & $++/+$ & 13 year PP mare \\
\hline D2 & SDFT, DDFT, SL & ++ & $+/-$ & $+/-$ & ND & 24 year PP male \\
\hline D3 & SDFT, DDFT & $+/-$ & $+/-$ & $+/-$ & ND & 14 year PP mare \\
\hline D4 & $\mathrm{LF}$ & $++/+$ & + & $+1-$ & ND & 17 year PP mare \\
\hline D5 & 2 SDFTs DDFT, SL & +++ & $\mathrm{N} / \mathrm{A}$ & ND & $+/-$ & fetus PP (of D4) \\
\hline D6 & DDFT, SL & +++ & $++/+$ & $++/+$ & $++/-$ & 18 mo TB female \\
\hline D7 & $\begin{array}{l}\text { DDFT, SL } \\
\text { SDFT }\end{array}$ & $\begin{array}{l}++/+ \\
+\end{array}$ & + & ND & ND & 20 year PP male \\
\hline $\mathrm{C} 1$ & SDFT, DDFT, SL & - & $\mathrm{N} / \mathrm{A}$ & $+/-$ & $+/-$ & 33 year PP stallion \\
\hline $\mathrm{C} 2$ & DDFT, SL & $+/-$ & $\mathrm{N} / \mathrm{A}$ & $+/-$ & $+/-$ & 8 year $\mathrm{QH}$ female \\
\hline $\mathrm{C} 3$ & DDFT, SL & $+1-$ & $\mathrm{N} / \mathrm{A}$ & $+1-$ & $+/-$ & 5 mo Percheron male \\
\hline C4 & SDFT, DDFT, SL & $++/+$ & $\mathrm{N} / \mathrm{A}$ & $+/-$ & ND & 31 year PP mare \\
\hline $\mathrm{C} 5$ & SDFT, DDFT, SL & $+/-$ & N/A & $+/-$ & ND & 32 year PP mare \\
\hline
\end{tabular}

H DSLD horse with many active foci or hypercellularity, limited PGs, D DSLD horse with mostly PGs and limited hypercellularity, C control horse, PP Peruvian Paso, QH quarter horse, TB thoroughbred, SDFT superficial deep digital flexor tendon, DDFT deep digital flexor tendon, $S L$ suspensory ligament, $R H$ right hind limb 


\section{Immunohistochemistry}

Standard hematoxylin and eosin staining of tissue sections was used for initial evaluation and diagnosis. Immunohistochemistry was performed on deparaffinized slides using a standard protocol. Endogenous peroxidase was quenched with $3 \% \mathrm{H}_{2} \mathrm{O}_{2}$ for $10 \mathrm{~min}$ at room temperature (RT). After washing with a buffer nonspecific sites were blocked with a universal blocking agent (Biogenex Laboratories, Fremont, CA, USA). Following antibodies were used: rabbit polyclonal antibody sc-146 (Santa Cruz Biotechnology, Santa Cruz, CA, USA) to detect TGF $\beta-1$ at dilution $1: 500$ for $2 \mathrm{~h}$ at $37^{\circ} \mathrm{C}$ or overnight at $4{ }^{\circ} \mathrm{C}$; antiBMP2 rabbit polyclonal antibody ab82511 (Abcam, Cambridge, UK) was used at dilution $1: 1000$ for $2 \mathrm{~h}$ at $37^{\circ} \mathrm{C}$ or overnight at $4{ }^{\circ} \mathrm{C}$, and rabbit polyclonal anti-glucuronic acid epimerase antibody (Novus Biologicals, Littleton, CO, USA) was used at 1:200 dilution for $1 \mathrm{~h}$ at RT. After incubation with a primary antibody the slides were incubated with secondary biotinylated antirabbit antibody for $2 \mathrm{~h}$ at RT before Avidin Biotin complex (Elite PK-6100 Standard kit) (Vector Burlingame, CA, USA) was applied for $1 \mathrm{~h}$ at RT. Antibody-antigen complexes were detected with 3,3'-diaminobenzidine (DAB) Peroxidase (HRP) Substrate Kit (with Nickel, SK-4100), also from Vector Laboratories. Slides were counterstained with hematoxylin. Primary antibodies were omitted in control slides. The staining was evaluated primarily for intensity and presence in fibroblasts or tenoblasts (from + to +++ ) rather than distribution and range in extracellular tissue because collagen and even more proteoglycans exhibit strong background staining.

\section{Results}

\section{Subjects}

Tendons and SLs were obtained from 21 horses from DSLD and from 5 control horses without DSLD (Table 1). The DSLD group was divided in two sub-groups, one consisting of 14 subjects $(\mathrm{H} 1-\mathrm{H} 14)$ with prominent hypercellular foci in their tendons and SLs, whereas tendons and SLs from 7 horses with prevalence of large proteoglycan collections were in included in the second sub-group (D1-D7. The horses ranged from a fetus (D5) to 33 years $(\mathrm{C} 1, \mathrm{C} 4$ and $\mathrm{C} 5))$ in age with both sexes and several breeds included (Table 1).

\section{Histopathology}

Normal tendons, especially those of adult horses, are not very cellular. They consist of thick collagen fibers with few tenocytes scattered within and among fibers. Bundles and fascicles are separated by septa of loose connective tissue containing fibroblasts, loose collagen fibers, adipose tissue and small blood vessels (Fig. 1a).
Proteoglycans are present in normal tendons and SLs in small amounts and are identifiable only with special stains, such as alcian blue. One of their roles is to regulate collagen fibrillogenesis [3]. As the basic histopathology of DSLD was described previously [3], we are pointing out only the major points relevant for this study. The hallmark of DSLD are inappropriate acellular foci or accumulations of proteoglycans in tendons and other connective tissues, especially SLs without any evidence of inflammation. Such deposits are clearly recognizable even with hematoxylin-eosin staining as blue to purple acellular material (Fig. 1b) and likely represent a more advanced stage of DSLD, particularly when cartilage metaplasia and occasional foci of calcification are present.

In addition, we have observed two types of increased cellularity in some cases of DSLD. The first type forms distinct foci in affected tendons and SLs, consisting of spindly, plump active fibroblasts/tenoblasts, sometimes arranged in whorls and containing small amounts of cytoplasmic proteoglycans (Fig. 1c). The foci were not associated with what we consider more established areas of DSLD (though they may co-exist in the same tendon) described above. However, even in the more advanced stage of DSLD increased cellularity was observed in septa separating bundles and fascicles of quite a few tendons and SLs (Fig. 1d), especially in younger horses. The cells were fibroblasts arranged in sheets. On occasion their cytoplasm also contained small amount of proteoglycans. Proliferation of small blood vessels containing proteoglycans in their media at times was noted as well (Fig. 1d).

\section{Presence of TGF $\beta-1$ and $B M P 2$}

Of the numerous growth factors participating in normal physiology of a tendon TGF $\beta-1$ is probably the most potent stimulant of synthesis of components of extracellular matrix, including type I collagen, the crucial structural constituent of tendons and, to lesser extent, SLs [15]. However, the presence of cartilaginous like material in DSLD-affected tendons and SLs indicates that other, chondrogenic growth factors might be instrumental in DSLD pathogenesis, leading to the accumulation of proteoglycan masses and cartilage metaplasia. BMP2 is one of such growth factors. TGF $\beta-1$ as a potent stimulator of collagen production should also be present in tendons and ligaments. Using immunohistochemistry we have identified only mild amount of TGF $\beta-1$ in both control and DSLD-affected tendon and ligaments (Table 1, Fig. 2a, b). This could be because collagens have long half life and so the turnover would be rather slow with no need for TGF $\beta-1$ to be very active and/or overexpressed. TGF $\beta-1$ was present in fibroblasts and tenocytes, but not in collagen fibers of both normal and DSLDaffected tissues or in the proteoglycan masses (Fig. 2a, 


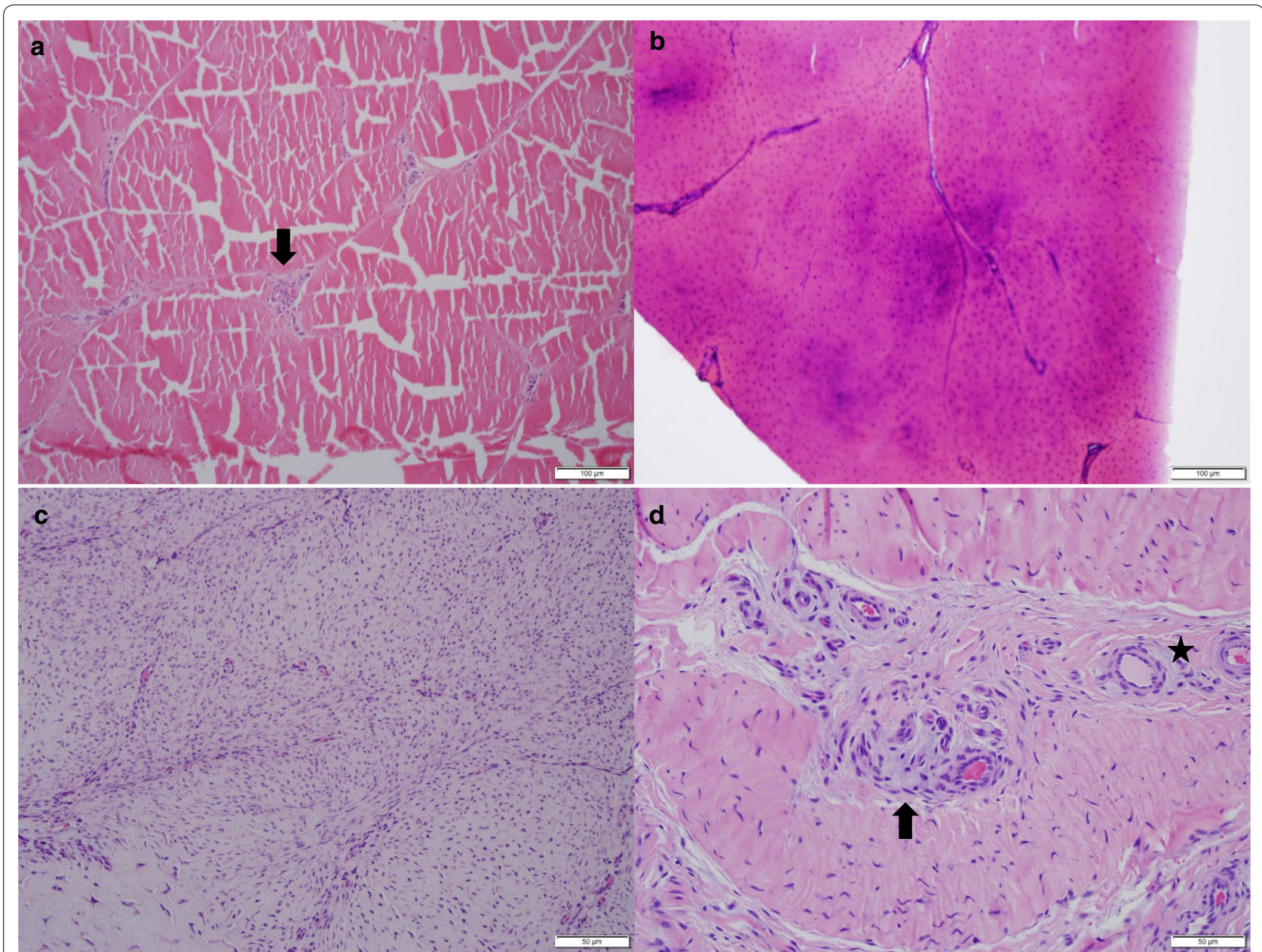

Fig. 1 Basic histopathology of DSLD lesions. a Histology of normal tendon shows bundles and fascicles separated by septa of less organized and somewhat loose connective tissue that contains fibroblasts, loose collagen fibers, adipose tissue and small blood vessels ( $\downarrow$ ). b A DSLD-affected tendon shows infiltration of proteoglycans (staining dark blue or purple) obscuring the normal architecture of the tendon. c Cellular lesions are visualized as distinct foci consisting of spindly active fibroblasts/tenoblasts, arranged in whorls. The presence of small amounts proteoglycans is limited to the cytoplasm of these cells. $\mathbf{d}$ Reveals an area of septum with increased number of fibroblasts containing small amount of proteoglycans $(\downarrow)$. Numerous small blood vessels are present as well $\left(^{*}\right)$. All four sections are stained with hematoxylin and eosin

b). Immunohistochemistry showed increased levels of BMP2 in cytoplasm of tenoblasts forming cellular foci and whorls in DSLD-affected tissues (Fig. 2d). The acellular areas or masses containing proteoglycans showed no or only little BMP2 staining, and if present staining for BMP2 was limited to cells surrounding proteoglycan mass (Fig. 2e). No or very little BMP2 was identified in the control tissues (Fig. 2f). One section of a tendon from C4 control horse revealed a small area where cells with normal morphology and arrangement stained moderately for BMP2.

Immunohistochemistry identified both TGF $\beta-1$ and BMP2 in tendon sheath and coverings like peritenon and epitenon in all examined samples in moderate amounts regardless of the presence of DSLD.

\section{Epimerase presence}

That dermatan sulfate epimerase was identified in both healthy and DSLD-affected tissues is not surprising as it is required for proper formation of dermatan sulfate, a GAG chain complexed with decorin protein core. Immunohistochemistry revealed only mild staining in tenoblasts of both normal and DSLD-affected tendons and SLs.

\section{Discussion}

Until recently, equine DSLD was considered to be a collagen disorder localized to SLs of distal limbs of horses. We have shown that not only it is a systemic disorder, but that the primary problem is an excessive accumulation of proteoglycans [3]. We have identified altered glycosylation of 

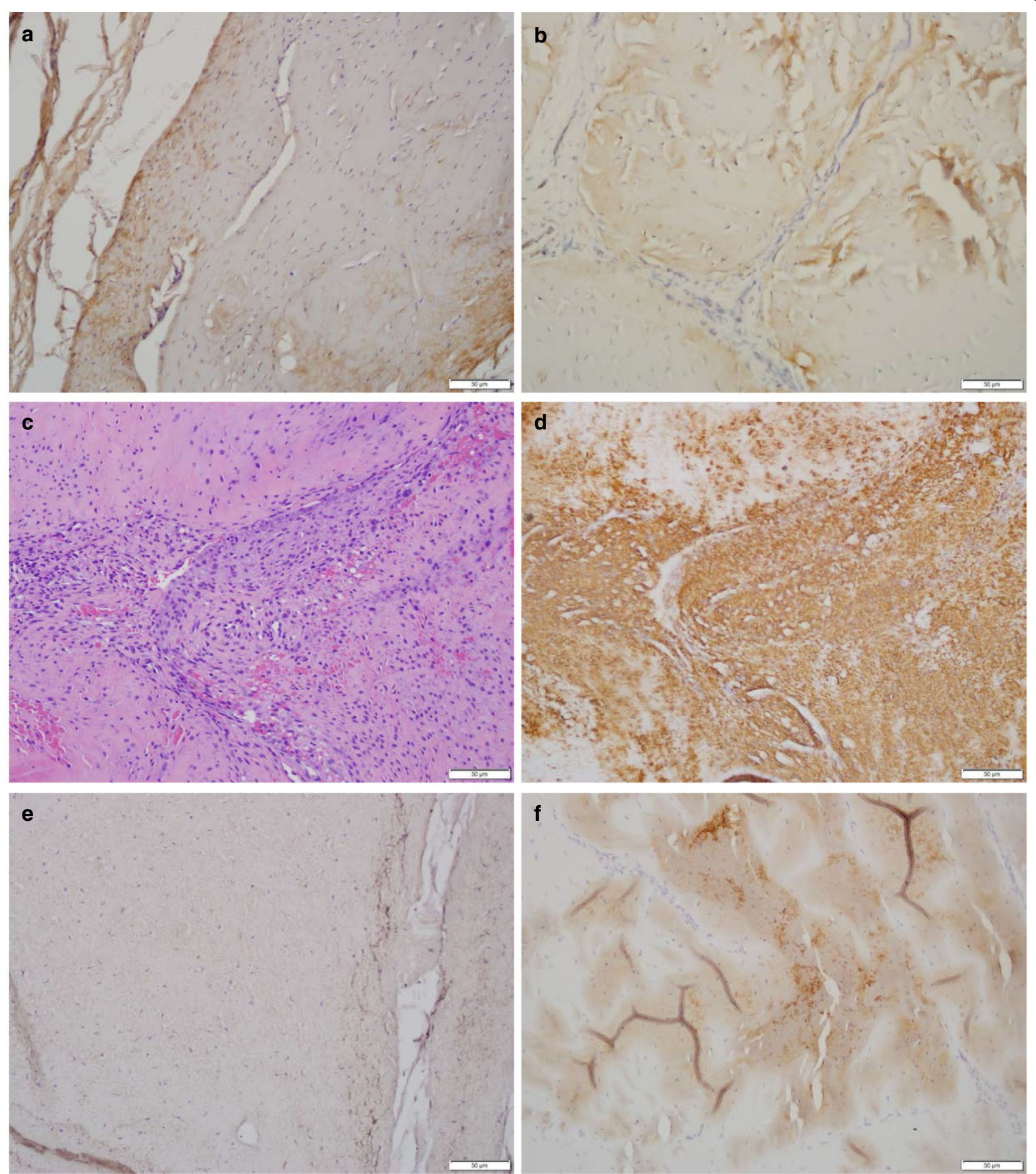

Fig. 2 Immunohistochemical visualization of TGF $\beta-1$ and BMP2. Using immunohistochemistry only little TGF $\beta$-1 was visualized in fibroblasts and tenoblasts, in both control (a) and DSLD-affected (b) tendons. c Reveals hematoxylin and eosin stained cellular foci and whorls in DSLD-affected tendons. $\mathbf{d}$ The cells in these foci immunostained strongly for BMP2. No or only little BMP2 was present in acellular areas or masses containing proteoglycans (e) and likewise no or very little BMP2 was identified in cells of the control tendons (f) 
decorin GAG chain in which chondroitin sulfate is substituted for the usual dermatan sulfate [5]. We hypothesized that changes in dermatan sulfate epimerase might be responsible for the abnormal substitution and that increased activity of a chondrogenic growth factor(s) may play roles in pathogenesis of DSLD [5]. To further elucidate this mechanism we have investigated the presence of BMP2, a chondrogenic growth factor, TGF $\beta-1$, a potent stimulator of collagen synthesis, and dermatan sulfate epimerase, an enzyme responsible for conversion glucuronic acid into iduronic acid during synthesis of dermatan/chondroitin sulfate chains in tendons and SLs collected from control and DSLD-affected horses. Our goal was to identify compounds important for the development of inappropriate proteoglycan accumulation and cartilage metaplasia in equine DSLD. The increased presence of BMP2 in cellular foci together with the presence of small amounts of proteoglycans in cytoplasm of these tenoblasts/fibroblasts suggests direct involvement of BMP2 in production of proteoglycans by DSLD-affected tenoblasts. The absence of BMP2 in masses of proteoglycans is not unexpected as these lesions are largely acellular and, in all likelihood, represent late development of DSLD where proteoglycans replace not only cells but also collagen fascicles and bundles.

Little or no BMP2 was found in control tendons and SLs because BMP2 stimulates the formation and proliferation of osteoblasts and chondrocytes rather than tenoblasts, and therefore it is usually not found in normal tendons and SLs. Finding high levels of BMP2 in DSLD tendon and SL tissues supports our hypothesis that the overproduction of proteoglycans could stem from an overabundance of at least one chondrogenic growth factor and confirms findings of BMPs in cases of human patellar tendinopathy characterized by cartilage formation [16]. In DSLD-affected tissues the level of immunostained TGF $\beta-1$ was surprisingly low. This is interesting because TGF $\beta-1$ is well known to have osteoblastic as well as chondrogenic effects on mesenchymal stem cells [15]. TGF $\beta-1$ is known to be present in tendons and ligaments, though its low level of expression in normal and DSLD-affected tendon cells/fibroblasts may be indicative of low metabolic turnover of collagen metabolism in the tendon and SL. This would be in agreement with a recent study showing a decrease in TGF $\beta-1$ signaling target gene expression in adipose-derived stromal fibroblasts in DSLD Peruvian Paso horses [17].

We did expect the levels of dermatan sulfate epimerase to be lower in DSLD tissues because our previous data have shown decreased conversion of glucuronic acid to iduronic acid, and thus for synthesis of chondroitin sulfate rather than dermatan sulfate, a process performed by dermatan sulfate epimerase [5]. However, a mutation in the epimerase gene in DSLD would not be detected by immunohistochemistry.

Therefore, we were not able to determine whether the enzyme is active or whether it underwent mutation in DSLD, and thus a change in activity rather than quantity.

In conclusion, we hypothesize that proteoglycan producing cells, most likely tenoblasts, form foci in active disease and are driven to produce excessive amounts of proteoglycans through secretion of BMP2, and perhaps of other chondrogenic growth factors during progression of DSLD.

\section{Limitations}

- Only immunohistochemistry was used.

- Poor results with in situ hybridization because of strong background staining of acellular proteoglycans.

- The use of antibodies to only a few molecules was due to budget limits.

\section{Abbreviations}

BMP: bone morphogenetic protein; DDFT: deep digital flexor tendon; DSLD: degenerative suspensory ligament desmitis; GAG: glycosaminoglycan; RT: room temperature; SDFT: superficial digital flexor tendon; SL: suspensory ligament; TGF $\beta$ : transforming growth factor $\beta$.

\section{Authors' contributions}

$J H$ developed the study concept and designed experiments. JH and MY wrote the manuscript. JZ, MY, OM and CAS performed immunohistochemistry. JH, MY, OM and CAS interpreted the immunohistochemistry data. POEM performed clinical examination of horses and nuchal biopsies. All authors contributed to discussion, read and reviewed the manuscript. All authors read and approved the final manuscript.

\section{Author details}

${ }^{1}$ Department of Pathology, College of Veterinary Medicine, The University of Georgia, Athens, GA 30602, USA. ${ }^{2}$ Department of Large Animal Medicine, College of Veterinary Medicine, The University of Georgia, Athens, GA 30602, USA. ${ }^{3}$ Department of Pathology, College of Veterinary Medicine, AU/UGA Medical Partnership, The University of Georgia, Athens, GA 30602, USA.

\section{Acknowledgements}

Not applicable.

\section{Competing financial interests}

The authors declare that they have no competing interests.

\section{Availability of data and materials}

All slides are stored in Dr. Halper's lab at The University of Georgia and are available from the corresponding author on reasonable request.

\section{Consent to publish}

Not applicable.

\section{Ethics approval and consent to participate}

All procedures and tissue collection have been approved by the Institutional Animal Care and Use Committee at The University of Georgia (IACUC\# A2016 11-020-R1).

\section{Funding}

This research was supported by the University of Georgia Research Foundation, University of Georgia Competitive Intramural Equine Program, Paso Peruano Europa, International Curly Horse Organization, Center for Undergraduate Research Opportunities Assistantship to M.Y., training grant awarded by 
the Tertiary Education Trust Fund, Federal University of Agriculture, Abeokuta, Nigeria to O.M., and private donations.

\section{Publisher's Note}

Springer Nature remains neutral with regard to jurisdictional claims in published maps and institutional affiliations.

Received: 27 July 2018 Accepted: 10 September 2018

Published online: 18 September 2018

\section{References}

1. Mero JL, Pool R (ed). Twenty cases of degenerative suspensory ligament desmitis in Peruvian Paso horses. In: Proceedings, annual convention of AAEP 2002; Orlando.

2. Young $\mathrm{JH}$. Degenerative suspensory ligament desmitis. Hoofcare Lameness. 1993;6:19.

3. Halper J, Kim B, Khan A, Yoon JH, Mueller PO. Degenerative suspensory ligament desmitis as a systemic disorder characterized by proteoglycan accumulation. BMC Vet Res. 2006;2:12.

4. Halper J, Khan A, Mueller POE. Degenerative suspensory ligament desmitis - a new reality. Pak Vet J. 2011;31(1):1-8.

5. Kim B, Yoon JH, Zhang J, Eric Mueller PO, Halper J. Glycan profiling of a defect in decorin glycosylation in equine systemic proteoglycan accumulation, a potential model of progeroid form of Ehlers-Danlos syndrome. Arch Biochem Biophys. 2010;501(2):221-31.

6. Danielson KG, Baribault H, Holmes DF, Graham H, Kadler KE, lozzo RV. Targeted disruption of decorin leads to abnormal collagen fibril morphology and skin fragility. J Cell Biol. 1997;136(3):729-43.

7. lozzo RV. The family of the small leucine-rich proteoglycans: key regulators of matrix assembly and cellular growth. Mol Biol Cell. 1997;32:141-74.
8. Iozzo RV, Schaefer L. Proteoglycan form and function: a comprehensive nomenclature of proteoglycans. Matrix Biol. 2015;42:11-55.

9. Scarfi S. Use of bone morphogenetic proteins in mesenchymal stem cell stimulation of cartilage and bone repair. World J Stem Cells. 2016:8:1-12.

10. Cicione C, Muiños-López E, Hermida-Gómez T, Fuentes-Boquete I, Díaz-Prado S, Blanco FJ. Alternative protocols to induce chondrogenic differentiation: transforming growth factor- $\beta$ superfamily. Cell Tissue Bank. 2015;15:195-207

11. Li J, Dong S. The signaling pathways involved in chondrocyte differentiation and hypertrophic differentiation. Stem Cells Int. 2016;2016:1-12.

12. Tiedemann $K$, Larsson T, Heinegård D, Malmström A. The glucuronyl C5-epimerase activity is the limiting factor in the dermatan sulfate biosynthesis. Arch Biochem Biophys. 2001;391:65-71.

13. Tiedemann K, Olander B, Eklund E, Todorova L, Bengtsson M, Maccarana $M$, et al. Regulation of the chondroitin/dermatan fine structure by transforming growth factor-beta1 through effects on polymer-modifying enzymes. Glycobiology. 2005;15(12):1277-85.

14. Maccarana M, Kalamajski S, Kongsgaard M, Magnusson SP, Oldberg A, Malmström A. Dermatan sulfate epimerase 1-deficient mice have reduced content and changed distribution of iduronic acids in dermatan sulfate and an altered collagen structure in skin. Mol Cell Biol. 2009:29:5517-28.

15. Halper J. Growth factors as active participants in carcinogenesis: a perspective. Vet Pathol. 2010;47(1):77-97.

16. Rui YF, Lui PP, Wong YM, Tan Q, Chan KM. BMP-2 stimulated non-tenogenic differentiation and promoted proteoglycan deposition of tendonderived stem cells (TDSCs) in vitro. J Orthop Res. 2013:31(5):746-53.

17. Luo W, Sandy J, Trella K, Gorski D, Gao S, Li J, et al. Degenerative suspensory ligament desmitis (DSLD) in Peruvian Paso horses is characterized by altered expression of TGF $\beta$ signaling components in adipose-derived stromal fibroblasts. PLoS ONE. 2006;11:e0167069.
Ready to submit your research? Choose BMC and benefit from:

- fast, convenient online submission

- thorough peer review by experienced researchers in your field

- rapid publication on acceptance

- support for research data, including large and complex data types

- gold Open Access which fosters wider collaboration and increased citations

- maximum visibility for your research: over $100 \mathrm{M}$ website views per year

At $\mathrm{BMC}$, research is always in progress.

Learn more biomedcentral.com/submissions 